eISSN 2444-7986

DOI: https://doi.org/10.14201/orl201674.14804

Artículo de revisión

\title{
EVIDENCIA Y RECOMENDACIÓN. ¿HARMONIC ULTRACISION OFRECE VENTAJAS EN TIROIDECTOMÍA?
}

\section{Evidence and recommendation. Does Harmonic Ultracision Focus offer any advantage in thyroidectomy?}

José Luis PARDAL-REFOYO

SACYL. Complejo Asistencial de Zamora. Servicio de Otorrinolaringología. Zamora. España.

Correspondencia: jlpardal@usal.es

Fecha de recepción: 18 de junio de 2016

Fecha de aceptación: 24 de junio de 2016

Fecha de Publicación: 27 de junio de 2016

Conflicto de intereses: Los autores declaran no tener conflictos de intereses

Imágenes: Los autores declaran haber obtenido las imágenes con el permiso de los pacientes

Política de derechos y autoarchivo: se permite el autoarchivo de la versión post-print (SHERPA/RoMEO)

Licencia CC BY-NC-ND. Licencia Creative Commons Atribución-NoComercial-SinDerivar 4.0 Internacional

๔ ) Universidad de Salamanca. Su comercialización está sujeta al permiso del editor

RESUMEN

Introducción y objetivo: En un paciente con patología de tiroides [paciente] sometido a tiroidectomía [intervención] la técnica realizada con Harmonic Ultracision ${ }^{\circledR}(\mathrm{HU})$ comparada con las técnicas convencionales (ligadura, electrocoagulación mono o bipolar y Ligasure $®$ ) [comparación] ¿es más segura [resultado]? Material y método: Búsqueda bibliográfica en las bases de datos PubMed, Cochrane Library y Scopus con la estrategia de búsqueda «(((((ultrasonic scalpel) OR harmonic)) AND thyroidectomy)) AND meta-analysis. Resultados: Nivel de evidencia: La evidencia de la ventaja del uso de HU en tiroidectomía frente a otras técnicas es alta respecto a la eliminación de las suturas, a la facilitación del acceso a espacios más reducidos, la reducción del tiempo quirúrgico, la reducción del sangrado intraoperatorio y postoperatorio, la reducción del hipoparatiroidismo transitorio, la reducción del dolor postoperatorio, la reducción de la estancia hospitalaria y reducción del coste. Conclusiones: Recomendación: La recomendación del uso de $\mathrm{HU}$ en tiroidectomía frente a otras alternativas (cirugía convencional / Ligasure $($ ) es débil a favor porque reduce la incidencia de parálisis laríngea y es fuerte a favor debido a que reduce el tiempo operatorio, reduce el sangrado intraoperatorio y postoperatorio, reduce el volumen drenado, reduce la incidencia de hipocalcemia, reduce la estancia hospitalaria y el coste. La recomendación del uso de HU en tiroidectomía es fuerte a favor.

PALABRAS CLAVE evidencia y recomendación; tiroidectomía; Harmonic; bisturí de ultrasonido; seguridad del paciente; Ligasure

SUMMARY Introduction and objective: In a patient with thyroid disease [patient] undergoing thyroidectomy [intervention] is the technique performed with Harmonic Ultracision Focus ${ }^{\circledR}(\mathrm{HU})$ compared with conventional techniques (ligation, mono or bipolar electrocoagulation and Ligasure $®$ ) [comparison] safer [outcome]? Material and Methods: Literature search in PubMed databases, Cochrane Library and Scopus with the search strategy "((((ultrasonic scalpel) OR harmonic)) AND thyroidectomy)) AND meta-analysis" was made. Results: Level of Evidence: Evidence of

(c) Ediciones Universidad de Salamanca / CC BY-NC-ND [237]

Rev. ORL, 2016, 7, 4, pp. 237-241 
the advantage of using $\mathrm{HU}$ in thyroidectomy compared to other techniques is high on disposal of sutures, facilitating access to smaller spaces, reducing surgical time, reducing intraoperative and postoperative bleeding, reducing of transient hypoparathyroidism, reducing postoperative pain, reducing hospital stay and reducing costs. Conclusions: Recommendation: The recommendation of the use of $\mathrm{HU}$ in thyroidectomy facing other alternatives (conventional surgery / Ligasure $($ ) is weak in favor because it reduces the incidence of laryngeal paralysis and is strongly in favor because it reduces operating time, intraoperative bleeding and postoperative drainage volumen. It also reduces the incidence of hypocalcemia, hospital staying and costs. The recommendation of the use of $\mathrm{HU}$ in thyroidectomy is strongly in favor.

KEYWORDS

evidence and recommendation; thyroidectomy; Harmonic; ultrasound scalpel; safety patient; Ligasure

\section{SITUACIÓN DEL TEMA}

La seguridad del paciente se define como el conjunto de medidas encaminadas a evitar daños innecesarios en el paciente durante el proceso asistencial. Estas están relacionadas con medidas técnicas y de gestión del proceso [1]. Los incidentes de seguridad en cirugía tiroidea se relacionan con la hemostasia, la función de los nervios laríngeos recurrentes, con los niveles de calcio y con la vía aérea [1]. Los incidentes de seguridad incrementan los riesgos para el paciente (muerte, secuelas), la instrumentación, la morbilidad, la estancia hospitalaria y los costes del proceso [1].

Tres son las técnicas de hemostasia tradicionalmente utilizadas en tiroidectomía: la técnica convencional con ligaduras y electrocoagulación mono o bipolar (a veces con clamps vasculares), LS y HU. La tecnología de la energía aplicada a la hemostasia en la cirugía ha supuesto un avance indudable. Instrumentos como Harmonic Ultracision $®(\mathrm{HU})$ mediante ultrasonidos (Ethicon Endosurgery) o Ligasure $®$ (LS) mediante energía eléctrica bipolar (Covidien) se vienen utilizando en tiroidectomía desde los años 90 del pasado siglo.

Harmonic $\circledR$ se basa en el principio piezoeléctrico y transmite ultrasonido desde el terminal a los tejidos a una frecuencia de $55500 \mathrm{~Hz}$ con un desplazamiento longitudinal variable entre 50 (corte más lento, mayor coagulación) y 100 $\mu \mathrm{m}$ (corte más rápido), generando una temperatura entre 34 y $80^{\circ} \mathrm{C}$. Harmonic $®$ ofrece ventajas iniciales intrínsecas: permite disección, corte y coagulación simultáneos, provoca mínimo daño tisular, no hay paso de corriente eléctrica a través del paciente y produce menos humo (produce un bioaerosol bifásico de agua y tejido con una pequeña proporción de humo sin acrilamida) [2].

El objetivo de este trabajo es revisar las ventajas de Harmonic Ultracision $®$ frente a otros sistemas de corte, coagulación y hemostasia en tiroidectomía.

\section{PREGUNTA CLÍNICA (PICO)}

En un paciente con patología de tiroides [paciente] sometido a tiroidectomía [intervención] la técnica realizada con Harmonic Ultracision ${ }^{\circledR}$ comparada con las técnicas convencionales (ligadura, electrocoagulación mono o bipolar y Ligasure $\left.{ }^{\circledR}\right)$ [comparación] ¿es más segura [resultado]?

\section{BÚSQUEDA BIBLIOGRÁFICA}

Se realizó una búsqueda bibliográfica sistemática en las bases de datos PubMed (https://www.ncbi.nlm.nih.gov/pubmed), Cochrane Library (http://www.bibliotecacochrane.com), y Scopus (https://www.scopus.com) con los descriptores y estrategias de búsqueda que se recogen en la figura 1.

La búsqueda se restringió al periodo 20102016 (a fecha de 30 de mayo de 2016) a metanálisis, en idioma inglés o español, tiroidectomía total mediante cirugía abierta (excluida MIVAT, cirugía endoscópica o robótica), pacientes adultos y que comparan la técnica con HU frente a la cirugía convencional (CC) incluido o no Ligasure (LS).

En la tabla 1 se resumen los resultados principales del análisis cualitativo de los artículos seleccionados.

Los resultados para evaluar la eficacia de una técnica frente a otras se establecen evaluando parámetros de gestión (tiempo operatorio, estancia hospitalaria), sangrado (sangrado intraoperatorio, volumen drenado en el postoperatorio), confort postoperatorio (dolor, vómitos) complicaciones postoperatorias (hematoma que precisa reintervención, hipoparatiroidismo transitorio y permanente, parálisis de nervio laríngeo recurrente transitoria y permanente, infección de la herida, seroma de la herida) o coste económico. 
Los resultados son difíciles de comparar debido a la escasez de ensayos clínicos aleatorizados que incrementa la utilización de los mismos ensayos en los metanálisis y a los sesgos que pueden producirse debido a la heterogeneidad de las muestras (tipos de patologías incluidas, experiencia del cirujano, comorbilidad).
Por otra parte hay un sesgo en el tiempo de implantación de las técnicas que hace que puedan no ser comparables unas con otras al cambiar los modelos de los terminales y generadores de energía.

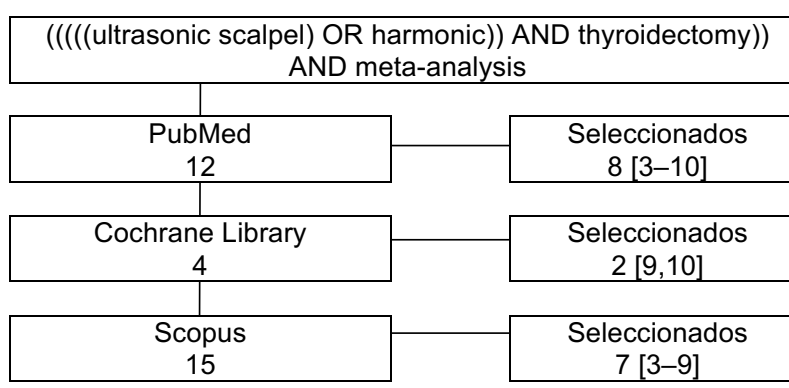

Selección para el

estudio cualitativo

Eliminados duplicados $8[3-10]$
31 resultados

Se eliminaron duplicados

Se excluyeron:

1 artículo en chino

3 artículos que no comparan las tres técnicas (convencional, HU, LS)

1 artículo relativo sólo al análisis de voz

2 cartas de réplica

1 artículo anterior a 2010

1 revisión sin metanálisis

Figura 1. ¿Harmonic Ultracision ofrece ventajas en tiroidectomía? Diagrama de flujo con la estrategia de búsqueda.

En términos generales, según la bibliografía revisada, HU ofrece ventajas puesto que simplifica el procedimiento quirúrgico, reduce pérdida de sangre, elimina la necesidad de los clips y las ligaduras de sutura, facilita la visibilidad por el menor sangrado intraoperatorio, se reduce significativamente el tiempo operatorio, reduce el dolor postoperatorio, reduce la tasa de hipocalcemia, facilita la cirugía en espacios reducidos y tiene menor coste debido al menor tiempo operatorio y menor estancia hospitalaria.
En este sentido HU ofrece mayor seguridad global en la realización de la tiroidectomía por lo que sería la tecnología de elección para esta técnica.

Son necesarios más ensayos clínicos aleatorizados que establezcan las ventajas de unos frente a otros sistemas de energía (puesto que parece comprobada la mayor eficacia y eficiencia de estos sistemas frente a la cirugía convencional basada en las ligaduras y electrocoagulación). 
¿HARMONIC ULTRACISION OFRECE VENTAJAS EN TIROIDECTOMÍA?

PARDAL REFOYO JL

Tabla 1. ¿Harmonic Ultracision Focus $₫$ ofrece ventajas en tiroidectomía? Tabla-resumen de los resultados de la búsqueda bibliográfica

\begin{tabular}{|c|c|c|c|}
\hline AUTOR, AÑO & $\begin{array}{l}\text { TIPO DE ESTUDIO } \\
\text { (PARÁMETROS) }\end{array}$ & COMPARACIÓN & $\begin{array}{l}\text { RESULTADOS PRINCIPALES } \\
\text { COMENTARIOS. VENTAJA DE HARMONIC® }\end{array}$ \\
\hline $\begin{array}{l}\text { Cannizzaro et } \\
\text { al. } 2016 \text { [3] }\end{array}$ & $\begin{array}{l}\text { Tiempo operatorio } \\
\text { Pérdida de sangre } \\
\text { Estancia hospitalaria } \\
\text { Dolor } \\
\text { Complicaciones }\end{array}$ & $\mathrm{HU}-\mathrm{CC}-\mathrm{LS}$ & $\begin{array}{l}\text { Reducción del tiempo quirúrgico } \\
\text { Menor pérdida de sangre, } \\
\text { Menor estancia hospitalaria, } \\
\text { No incrementa el dolor y las complicaciones }\end{array}$ \\
\hline $\begin{array}{l}\text { Cheng et al. } \\
2016[4]\end{array}$ & Coste & $\mathrm{HU}-\mathrm{CC}-\mathrm{LS}$ & $\begin{array}{l}\text { Menor coste (reduce los costes en un } 10 \% \text { respecto } \\
\text { a otras técnicas debido a la reducción del tiempo } \\
\text { operatorio. }\end{array}$ \\
\hline $\begin{array}{l}\text { Revelli et al. } \\
2016 \text { [5] }\end{array}$ & $\begin{array}{l}\text { Tiempo operatorio } \\
\text { Estancia hospitalaria } \\
\text { Sangrado intraoperatorio } \\
\text { Volumen drenado postope- } \\
\text { ratorio } \\
\text { Complicaciones de la he- } \\
\text { rida (infección y seroma) } \\
\text { Hipocalcemia postoperato- } \\
\text { ria (T/P) } \\
\text { Parálisis NLR (T/P) } \\
\text { Dolor postoperatorio }\end{array}$ & $\mathrm{HU}-\mathrm{CC}$ & $\begin{array}{l}\text { Simplifica el procedimiento /elimina la necesidad de } \\
\text { clips y ligaduras de sutura } \\
\text { Reduce significativamente el tiempo operatorio, } \\
\text { Reduce dolor postoperatorio } \\
\text { Reduce pérdida de sangre } \\
\text { Reduce la hipocalcemia } \\
\text { Facilita la cirugía en espacios reducidos al facilitar } \\
\text { la visibilidad por el menor sangrado intraoperatorio } \\
\text { Menor coste: menor tiempo, menor estancia. }\end{array}$ \\
\hline $\begin{array}{l}\text { Cheng et al. } \\
2015 \text { [6] }\end{array}$ & $\begin{array}{l}\text { Tiempo operatorio } \\
\text { Sangrado intraoperatorio } \\
\text { Dolor postoperatorio } \\
\text { Estancia } \\
\text { Volumen drenado } \\
\text { Hemorragia con reopera- } \\
\text { ción } \\
\text { Hipocalcemia } \\
\text { Parálisis NLR } \\
\text { Seroma de la herida }\end{array}$ & $\mathrm{HU}-\mathrm{CC}$ & $\begin{array}{l}\text { Reduce el tiempo operatorio, el sangrado intraope- } \\
\text { ratorio, el drenaje postoperatorio, el dolor y la es- } \\
\text { tancia. } \\
\text { El nuevo HU Focus tiene menor efecto térmico y es } \\
\text { más preciso. }\end{array}$ \\
\hline $\begin{array}{l}\text { Contin et al. } \\
2013[7]\end{array}$ & $\begin{array}{l}\text { Mortalidad } \\
\text { Sangrado intraoperatorio } \\
\text { Estancia } \\
\text { Parálisis NLR (T/P) } \\
\text { Hipocalcemia (T/P) } \\
\text { Sangrado postoperatorio } \\
\text { con reintervención } \\
\text { Hematoma / seroma } \\
\text { Reintervención } \\
\text { Infección de la herida }\end{array}$ & HU - CC - LS & $\begin{array}{l}\text { Menor tiempo operatorio. } \\
\text { La morbilidad postoperatoria no se ve afectada. } \\
\text { Puede ser útil en centros con alto volumen de inter- } \\
\text { venciones. } \\
\text { HU reduce el sangrado intra y postoperatorio sólo } \\
\text { frente a CC. } \\
\text { No hay diferencia en los otros parámetros o grupos. }\end{array}$ \\
\hline $\begin{array}{l}\text { Garas et al. } \\
2013[8]\end{array}$ & $\begin{array}{l}\text { Hipoparatiroidismo } \\
\text { Parálisis NLR (T/P) } \\
\text { Tiempo operatorio } \\
\text { Sangrado } \\
\text { Sangrado postoperatorio } \\
\text { Estancia hospitalaria } \\
\text { Coste }\end{array}$ & $\begin{array}{l}\mathrm{HU}-\mathrm{LS} \\
\mathrm{H}-\mathrm{CC}\end{array}$ & $\begin{array}{l}\text { Menor hipoparatiroidismo } \\
\text { Menor sangrado intraoperatorio y drenado } \\
\text { Menor tiempo quirúrgico } \\
\text { Menor estancia hospitalaria } \\
\text { Más parálisis laríngea con HU (no significativa) } \\
\text { Dos parámetros difíciles de evaluar: la experiencia } \\
\text { del cirujano y el volumen } \\
\text { No hay evidencia de coste-efectividad que orienten } \\
\text { por uno u otro dispositivo }\end{array}$ \\
\hline $\begin{array}{l}\text { Ecker et al. } \\
2010[9]\end{array}$ & $\begin{array}{l}\text { Tiempo operatorio } \\
\text { Sangrado intraoperatorio / } \\
\text { Drenaje } \\
\text { Complicaciones (parálisis } \\
\text { de NLR, disfonía, hipocal- } \\
\text { cemia, hematoma, seroma) } \\
\text { Dolor postoperatorio } \\
\text { Estancia }\end{array}$ & $\mathrm{HU}-\mathrm{CC}$ & $\begin{array}{l}\text { Menor tiempo quirúrgico, menor sangrado, menor } \\
\text { dolor postoperatorio, menor estancia. } \\
\text { Impacto económico. }\end{array}$ \\
\hline $\begin{array}{l}\text { Melck y Wise- } \\
\text { man, } 2010[10]\end{array}$ & $\begin{array}{l}\text { Tiempo quirúrgico } \\
\text { Parálisis NLR } \\
\text { Hipocalcemia }\end{array}$ & $\mathrm{HU}-\mathrm{CC}$ & $\begin{array}{l}\text { HU reduce el tiempo operatorio } \\
\text { Menor tasa de hipoparatiroidismo transitorio } \\
\text { Sin diferencia respecto a la parálisis de NLR }\end{array}$ \\
\hline
\end{tabular}

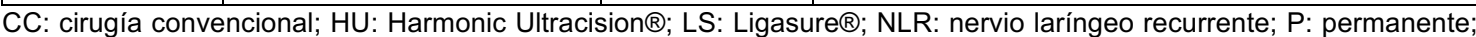
$\mathrm{T}$ : transitoria 
RESUMEN DE EVIDENCIA (GRADE)

$\mathrm{La}$ evidencia de la ventaja del uso de $\mathrm{HU}$ en tiroidectomía frente a otras técnicas es alta respecto a la eliminación de las suturas, a la facilitación del acceso a espacios más reducidos y a la reducción del tiempo quirúrgico, del sangrado intraoperatorio y postoperatorio, del hipoparatiroidismo transitorio, del dolor postoperatorio, de la estancia hospitalaria y del coste. Algunos parámetros no mejoran significativamente pero tampoco empeoran los resultados con HU por lo que la evidencia es baja respecto a la reducción de la parálisis laríngea o a la tasa de hipoparatiroidismo permanente.

\section{RECOMENDACIONES (GRADE)}

La recomendación del uso de $\mathrm{HU}$ en tiroidectomía frente a otras alternativas (cirugía convencional / Ligasure ${ }^{\circledR}$ ) es débil a favor porque reduce la incidencia de parálisis laríngea y es fuerte a favor debido a que reduce el tiempo operatorio, reduce el sangrado intraoperatorio y postoperatorio, reduce el volumen drenado, reduce la incidencia de hipocalcemia, reduce la estancia hospitalaria y el coste.

La recomendación del uso de $\mathrm{HU}$ en tiroidectomía es fuerte a favor.

\section{BIBLIOGRAFÍA}

1. Pardal Refoyo J. Aspectos prácticos para la mejora de la seguridad del paciente en cirugía de tiroides y paratiroides. Rev Soc Otorrinolaringol Castilla Leon Cantab La Rioja. 2015;6:S50-72. Disponible en: http://hdl.handle.net/10366/125711. [Citado el 18 de junio de 2016].

2. Krones CJ, Conze J, Hoelzl F, Stumpf M, Klinge $\mathrm{U}$, Moeller $\mathrm{M}$, et al. Chemical composition of surgical smoke produced by electrocautery, harmonic scalpel and argon beaming - a short study. Eur. Surg. 2007;39:118-21.

3. Cannizzaro MA, Borzì L, Lo Bianco S, Okatyeva V, Cavallaro A, Buffone A. Comparison between Focus Harmonic scalpel and other hemostatic techniques in open thyroidectomy: A systematic re-view and metaanalysis. Head Neck. 2016;38(10):1571-8.
4. Cheng $\mathrm{H}$, Soleas IM, Ferko NC, Cameron CG, Clymer JW, Amaral JF. Hospital costs associated with thyroidectomy performed with a Harmonic device compared to conventional techniques: a systematic review and meta-analysis. J. Med. Econ. 2016;19(8):750-8.

5. Revelli L, Damiani G, Bianchi CBNA, Vanella $S$, Ricciardi $W$, Raffaelli $M$, et al. Complications in thyroid surgery. Harmonic Scalpel, Harmonic Focus versus Conventional Hemostasis: A meta-analysis. Int. J. Surg. Lond. Engl. 2016;28 (Suppl 1):S22-32.

6. Cheng H, Soleas I, Ferko NC, Clymer JW, Amaral JF. A systematic review and metaanalysis of Harmonic Focus in thyroidectomy compared to conventional techniques. Thyroid Res. 2015;8:15.

7. Contin $\mathrm{P}$, Gooßen K, Grummich K, Jensen K, Schmitz-Winnenthal $H$, Büchler $M W$, et al. ENERgized vessel sealing systems versus CONventional hemostasis techniques in thyroid surgery--the ENERCON systematic review and network meta-analysis. Langenbecks Arch. Surg. 2013;398:1039-56.

8. Garas G, Okabayashi K, Ashrafian H, Shetty K, Palazzo F, Tolley N, et al. Which hemostatic device in thyroid surgery? A network meta-analysis of surgical technologies. Thyroid. 2013;23:1138-50.

9. Ecker T, Carvalho AL, Choe J-H, Walosek $G$, Preuss KJ. Hemostasis in thyroid surgery: harmonic scalpel versus other techniques--a meta-analysis. Otolaryngol Head Neck Surg. 2010;143:17-25.

10. Melck AL, Wiseman SM. Harmonic scalpel compared to conventional hemostasis in thyroid surgery: a meta-analysis of randomized clinical trials. Int. J Surg Oncol 2010;2010:396079 